\title{
CURRENT TRENDS AND TREATMENTS IN DIABETIC RETINOPATHY
}

\author{
FIROZ MV*, VISHAL GUPTA N, SANDEEP KANNA
}

Department of Pharmaceutics, JSS College of Pharmacy, JSS Academy of Higher Education and Research, Mysore, Karnataka, India. Email: firoz.mv3@gmail.com

Received: 26 April 2019, Revised and Accepted: 24 May 2019

ABSTRACT

The drastically increasing issues of the disease scenario currently are with different types of diabetes all over the world. It has been reported, approximately 592 million are suffering from the disease throughout the world. It affects differently in different patients with the disease. There have been reports that it is affected differently and also has different side effects. It is also been reported that diabetes mellitus leads to the cause of diabetic retinopathy (DR) and also diabetic macular edema. It is considered as one of the most common causes which is linked to DR. DR has been considered as one of the most important causes for the loss of vision or impaired vision. The drugs show different types of incompatibility such as toxicity, solubility issues, aggregation, and chemical degradation these can be improved by applying several methods. DR is classified according to "Airlie House" into different categories and based on different strategies and consideration. It was found that DR is the main cause for vision loss and also there no much strategies for development of new treatment. The treatment involved is laser photocoagulation and vitrectomy, among these the effective treatment, was found to be laser photocoagulation. This is mainly characterized as proliferative and non-proliferative DR. Different therapeutic agents have been taken for the study these includes vascular endothelial growth factor, renin-angiotensin system inhibitors and nonsteroidal anti-inflammatory drugs, they are certainly different interventions for the treatment, they are nanotechnology and liposome. Nanotechnology applied is the most effective and also acceptable way of treatment.

Keywords: Diabetic macular edema, Diabetic retinopathy, Nonsteroidal anti-inflammatory, Drugs nanotechnology.

(C) 2019 The Authors. Published by Innovare Academic Sciences Pvt Ltd. This is an open access article under the CC BY license (http://creativecommons. org/licenses/by/4. 0/) DOI: http://dx.doi.org/10.22159/ajpcr.2019.v12i7.33774

\section{INTRODUCTION}

It is known from the reports that there are more than 592 million people all over the world who are suffering from diabetes by 2035 [1]. This is related to an increase in the level of glucose in the blood, which is also termed as hyperglycemia. Diabetes mellitus (DM) is a diverse assembly of metabolic syndromes considered by chronic hyperglycemia, i.e.persistent increased blood glucose level [2]. DM is a key cause in worldwide healthcare systems and strongly linked with some main health risk factors [3].

DM is mainly divided into different types based on its chronic conditions mentioned below as:

1. Type 1 - It needs to be identified early in adults and children and is characterized by the deficient in insulin production. Due to this, the islet beta cells are destroyed, and it is mainly characterized by auto-immune deficiency

2. Type 2 - In nearly $90-95 \%$ of most patients, it is characterized by the insulin production and also the resistance of the body toward insulin synthesis, this leads to the increase in levels of glucose and affects the liver and adipose tissue

3. The other form of diabetes except those two are gestational diabetes, these are usually seen in pregnant women with the slight increase in glucose level this is mainly due to the interaction of placental hormones with insulin receptor resulting in the unmanageable or uncontrollable level of glucose in the body [4].

DM leads to the cause of diabetic retinopathy (DR) [5]. From various consideration, ocular diabetic includes ocular neuropathy, uveitis, cataract, and different types of diabetes. DR has been considered as one of the most important causes for the loss of vision or impaired vision [6].

Recent advances in surgeries and medical treatments allow diabetic patients who have long suffered from devastating complications to have a better quality of life (QOL). However, DR still remains one of the leading causes of blindness [7]. Loss of vision caused by DR is happening by different mechanisms. The loss of central vision may also be due to macular edema [8].

Pathology of DR is characterized by several methods involving biochemical and also vascular changes some of them are mentioned as, the lasting hyperglycemic condition leading to dysfunction of vascular endothelial cells, which is due to the accumulation of oxidative stress, advanced glycan end-products, sorbitol and inflammation, and renin-angiotensin systemic (RAS) upregulation. Then later, it is the ischemia in of the retina occurs and provokes diabetic macular edema (DME), vitreous hemorrhages and retinal detachment, and in a later stage, it induces retinal dysfunction [9].

DME is one among the spectrum which is associated with DR; these results from the hyperglycemic state and also the other metabolic changes. DME is been identified by the accumulation of extracellular fluid (cerebrospinal fluid) in the retina; it occurs once the blood-retinal barrier (BRB) is broken down which is caused due to the dilated hyperpermeable capillaries and microaneurysms [10]. There are no reported approved drug therapies for DME in Europe or the US. The primary treatments are laser photocoagulation and vitrectomy; however, treatment is mostly ineffective in all patients or physicians are looking for new treatment options [11].

The glucose uptake by the body is blocked, which results in the hormone deficiency, the constant hyperglycemia in the body usually leads to many chronic effects, some of them can be a micro or a macro effect to the body such as heart disease, ischemia, stroke, and other heart-related diseases. Lasting hyperglycemia affects the small blood vessels; it damages the ocular renal artery and also the nervous system. Due to these reasons, it is relevant to maintain the increased blood glucose level and also to prevent neuropathy, nephropathy, and retinopathy [4].

To control the side effects such as retinopathy, different methods have been followed, which are innovative ideas from nanotechnology-based 
nanoparticles, which have been addressed in the review. The use of nanoparticles for different clinical purpose in the field of medicine is called nanomedicine. The size of nanoparticles should be (1-1000 nm), which are helpful nowadays to prevent and also treatment most of the diseases [12].

The number of effects shown by the drugs is mentioned below as:

1. Solvents having low solubility

2. High toxicity

3. To exhibit the therapeutic effect

4. Aggregation of particles

5. Chemical and enzymatic degradation of drug molecules

6. The half-time of the drug is reduced.

Due to this, some of the limitations can be overcome by loading the drug into the nanoparticles for:

1. Targeted drug delivery at the specific site

2. Sustained drug delivery systems

3. The delivery of drug can be improved both for bimolecular drugs and water-insoluble drugs

4. Side effects can be reduced where the toxicological reactions can be reduced.

In one of the studies conducted in the United States showed that people with 20-74 years showed blindness which is due to the DM which is a leading complication of DR [13].

\section{DAIBETIC RETINOPATHY}

DR that probably affects most of the patients with long-lasting DM. Intraretinal hemorrhage and microaneurysms are visualized with the early lesions which are generally noticed [14].

The group of experts in 1968 of Airlie House, Virginia classified DR; they used the standard retinal photographic field as benchmarks. The extension of "modified Airlie House classification" which has been used by different people for the Early Treatment of Diabetic Retinopathy Study (ETDRS), it has 13 defined complex levels of DR these ranges from no retinopathy that is from (level 10) to the cause of vitreous hemorrhage that is till (level 85) [15].

DR is the main reason for the blindness between a different age group in western countries. It is one of the exponential growing diseases in under-developed countries also. Patients having DR for much more reason are prone to blindness. Advanced DR and macular edema (clinically significant) and also lead to vision loss [16]

DR is a silent killing disease; they come in light only in the stage where final treatment of disease becomes difficult in most of the cases. It can be treated effectively at the early stages with regular screening. Unconditioned screening is also required so that the user gets decreased as expenditure in this type of process is a bit high. The cost expenditure is also reduced, detection using a photograph of the retina was automized using different ways such as a digital way of capturing the image and also image processing techniques. Blood vessels at the time of DR which are helpful to strengthen the retina start with leaking fluid and blood, which leads to visual marks known as lesions that look like microaneurysms, hemorrhage's, hard exudates, cotton wool spots, and blood vessel area [17].

In the recent scenario, research has progressively focused on identifying the signs in the neuroretina degeneration of diabetes. Corneal confocal microscopy (CCM) is been used to identify $[18,19]$. The selected participants of neuronal degeneration in diabetes without a clinical sign of DR can also be partitioned into different group of participants who may be or may not be suffering from DR. In the study, they have mentioned and also examined the usage of optical coherence tomography for knowing the thickness of retina as a CCM derived corneal nerve fiber length and any other different types of risk factors that are predicted from the 4 years of development and also worsening the case of disease in several individuals who are suffering from Type-1 diabetes [20].

\section{ASSESSMENT IN CASE OF DIABETIC RETINOPATHY}

The photograph of the retina was taken using a standard protocol. Once the pupil dilation takes place, retinal image of both the eyes was obtained using the digital retinal camera (Canon CR-DGi with a 10-D SLR back; Canon, Tokyo, Japan) which was thus centered on the optic disc to study the (ETDRS standard field 1) and the other were centered on the fovea (ETDRS standard field 2) [21].

Topmost educated persons in this field analyzed the change of the image quality in case of DR. DR was also observe to be present if any distinct lesions were seen by the ETRDS extremity scale showed on the hemorrhages, microaneurysms, cotton wool spots, hard blood vessels, venous bleeding, and new blood vessels [22]. Both the eyes, the retina has been examined for the function of DR, which has been numbered based on the above-mentioned classification into specific levels [23].

DME has also been noted for the hard exhumes which happens due to the presences of microaneurysms and a blot of hemorrhage with the disc diameter from the foveal center or the presence of focal coagulation which shows scars on macular areas. The presence of severe non-proliferative DR (NPDR), PDR or DME based on the eye disease prevalence, the research group was defined as vision-threatening DR [24].

\section{DIFFERENT TYPES OF TREATMENT}

It is done by laser treatment which is not generally given to patients with NPDR, due to the assignment of which gives brief information on health technology assessment. In this study, they pose a question on whether the involvement of pan-retinal laser treatment before the disease starts or which may be during the preproliferative stage, i.e.(Level R2), which may be more useful to prevent the terms of vision loss. Pan-retinal photocoagulation is also known as scatter photocoagulation.

The treatment is considered for DR, for that three issues to be considered:

- Treatment underwent without the risk of visual loss

- The vision loss risk during the treatment

- The treatment which leads to side effects. This causes laser treatment which knocks down the process that leads to loss of peripheral vision to maintain the central vision [25].

Treatment of this disease is based on the reports obtained from the ETDRS and DRS [26]. Less number of a non-randomized study conducted for laser treatment reported that in people with Type-1 diabetes at the NPDR stage which causes loss of vision are compared to treatment with waiting PDR stage, but the cause of this has no change and did not reach the statistical consequences [27]. The study was conducted by the Swedish Stenkula using the xenon arc photocoagulation which has mentioned and also reported to benefit the treating of severe NPDR in the clinical study conducted for PRP with one of the randomized treatments [28]

There are no approved drug therapies for DME in Europe or the US. The primary treatments are laser photocoagulation and vitrectomy, as mentioned above [11]. Laser photocoagulation is the present treatment for DME, and it has been shown to reduce the incidence of some of the cases with vision loss [29]. Photocoagulation can also have severe adverse effects, including pain during treatment, short- and long-term visual loss in many cases, restriction of the visual fields and night blindness.

Vitrectomy includes the removal of the vitreous and also with the required change with a fluid or gas, it is designated when there is fractional retinal detachment and also the vitreous hemorrhage. This may be of value in the treatment of DME that is caused by the resistance of the vitreous on the macula [11]. 


\section{PATHOGENESIS}

DR is considered based on multiple factors, which are the biochemical changes, inflammatory process and also the genetic factors. Hyperglycemia leads to increased activation and also the regulation of vascular endothelial growth factor (VEGF), protein kinase, growth factors, oxidative stress, and other related diseases and the pathways [10].

\section{DME}

DME is considered as the vascular leakage and the BRB breakdown. It is divided into focal or diffuse subtypes that can occur in other settings such as vitreous resistance or the epiretinal membrane on the retina. They are also involved in the retinal thickening and are subdivided into different methodologies, including stereo photographs and clinical examination [30].

\section{PDR}

Disease progression in case of DR and capillary nonperfusion can also lead to ischemia, which starts at the peripheral region and slightly move toward the central region of the macula and then it moves toward the nerve [31]. The increased development of new blood vessels and fibrovascular proliferation which causes ischemia, VEGF and other proliferative factors that are released to the retina [32]. NPDR

It causes thickening of the basement membrane (BM), loss of pericytes, intraretinal hemorrhages, venous bleeding, dilation of the venous, capillary non-perfusion, microaneurysms, and intraretinal microvascular abnormalities. The BM capillary thickening is also seen in collagen and laminin in the recently developed studies [33]. This type of abnormality is also caused and it affects the capillary for the autoregulation and also the involvement of proteins and also residing pericytes [34]. The loss of pericytes is monitored as pathological changes that occur in DR, the loss of which leads to change in the microvascular auto regulation and the further proliferation of endothelial cells [35].

Nanotechnology-based ocular drug delivery system is one of the biggest challenges and also this route notices with different constraints that are usually seen in the pharmaceutical background. The drug delivery by this route is been restricted to the study of anatomy and the physiology of the eye. Nanoparticles are to be a leading take over in the upcoming systems, which are usually recognized with the very low bioavailability $(5 \%-10 \%)$ of the administered drugs [36].

DR needs to be detected by both the systemic and also by ocular aspects; it is an ocular disease which has to be managed by ophthalmologist or endocrinologist [37]. The treatment needs to be included in the management of hyperglycemia values, the serum lipids, and blood pressure [12]. For improvement, it is usually done by the combination of different strategies as there is no other treatment for the DR, but these can retard and reduce some of the symptoms avoiding the blindness early. This is done by the current and traditional strategies which can be managed by laser surgery, pharmacological therapy, and vitrectomy [38].

DR can be prevented by monitoring and also controlling the hyperglycemia and blood pressure as per the study conducted in the United Kingdom for diabetic control and the complications trail [39]. The study showed that intensive glycemic control leads to a proliferation of type- 1 and type- 1 diabetes [40]. There are certain incidences and progression of DR that significantly reduces the blood pressure [41].

DR includes pan-retinal photocoagulation, focal laser photocoagulation, and surgical vitrectomy. The pan-retinal photocoagulation includes hundreds of laser burns which affect the retina and also causes the quantity of ischemic retina, which is known as angiogenesis. It is considered as a milestone in the treatment of different DR study conducted proved that the risk decreases the loss of vision in $50 \%$ of patients with severe DR [42].
The laser photocoagulation is a point in patients with clinically notable of macular edema and is mainly focused on the microaneurysms reducing the plasma leakage which is increased due to the increased swelling in the intraretinal [43]. The vitrectomy deals with the surgical way of removing the fibrovascular, blood, and vitreous tissues of the body. DR which is associated is growing with the extreme vitreous hemorrhage and also associated with the transaction of the macula. The different observation is carried out to restore the vision and also vitrectomy in different patients with PDR [44].

\section{PATHOGENESIS IN CASE OF DIFFERENT DIABETIC RETINOPATHY}

There are different processes to know the results of diabetes in case of retinopathy with the retinal microvasculature, which is of supreme importance. The mechanism of these type is multifactorial, which leads to hyperglycemia activating polyol pathway, the fabrication of advanced glycation oxidative stress and transcription pathway [14].

The one of the major and an important case of DR which causes vascular malformation, and it appears anywhere between the nerve fiber layer and also the outer plexiform layer. The DR leads to the microscopical evaluation of the retinal vessels with a reduction in the retinal vessels as a reduction in the pericytes which surrounds the endothelial cells with the injury in the early stages of DR [45].

\section{EPIDEMIOLOGY}

Blindness is a common cause for DR, a study conducted in the United States showed the identification of visual activity in different people of aged 20-74-year-old people and over 50000 new cases of retinal macular edema have been occurred regularly as per reports [46]. The patients with a convenient way of treating the disease remain untreated due to some of these reasons [47].

DR is an extremely treated type of diabetes with a clear-cut advancement of the diabetes control and complications trial [48]. Different UK Prospective Diabetes Study shows different results related to the patients suffering from type-2 diabetes [40].

\section{TO INITIATE PHARMACOLOGIC THERAPY IN DIABETIC RETINOPATHY}

There is different treatment in the photocoagulation of the physicians for the center threatening DME. The laser therapy which causes an enduring effect with the comprehension, but it is the best way involving DME 20/32 which is not known. Some physicians will treat symptomatic patients with center-involving DME and excellent VA with anti-VEGF drugs despite the off-label indication [49].

Recent studies have been focused on the pan-retinal photocoagulation focused on the golden standard of well-known growing therapy in PDR [43]. There is pan-retinal photocoagulation which leads to vision loss and also destruction and some of the possible side effects that are caused due to the vitreous hemorrhage and also the macular edema these are especially concerned with the surgical interventions and also the laser therapy which are responsible for the high risk [50].

There are different types of study that has been considered and shows about $25-45 \%$ of disease which can be treated with increased macular edema and the disturbances in the eyes [51]. The intravitreal injection of the anti-VEGF of the drug which has been considered with the choice to PDR treatment [52].

The damage to the eye is different, which may be generic or acquired. In these, the eye can be classified into two different types, namely:

1. The anterior segment

2. The posterior segment.

Some of the different parts of the eye such as cornea, iris pupil, aqueous humor, and the lens make up the anterior segment of the eye. The some of the other parts, namely retina, choroid, macula, vitreous humor, and 
optic nerve, forms the posterior part, which is also considered for the study. The larger part is the retina which is considered for the study, where the nerve receives the light that creates the impulses that are transmitted from the optic nerve to the brain whereas macula is the smallest region which is light sensitive [53].

The abnormalities or any other malfunction which occur in the ocular tissue has a very big impact on the patient's QOL. One of the important causes for the retinal disease which includes DR and age-related macular degeneration. Future development in the understanding of the molecular mechanism in the few decades is used in the treatment of ocular pathogenesis has been leading advancement [54].

There are different methods for the development of intravitreal injections for the administration of different drugs and its formulation, but this always shows different associated complications. The efficacy of it can be increased by improving the patient's compliances and relation with ongoing research to make the availability and feasibility of the drug available for different forms of delivery such as periocular and systemic.

The effective and convenient way for the delivery of the drug to different region and areas using innovative ideas. The anatomy and the constraints of the eye which is taken into primary consideration in this study. There are several examples that show the management of the nanoparticles in the management of the different ocular diseases that have a perspective, challenges, and also opportunities for the development of retinal drug delivery [53].

There are different sized nanoparticles that are considered as the fast-growing and effective in the treatment of the different disease in pharmaceutical science [55]. The main aim was to develop the best soluble drug which is poorly water-soluble to formulate in the treatment of retina which helps in the transport, uptake, of the drugs from the biological barriers which lead to the prolonged release of drug and also prevent the drug from degradation. There are different synthetic polymer includes dendrimers, hydrogels, liposomes, proteins, and inorganic compounds [56].

There are different sized particles, and the surface properties of different drug molecules have been taken, which exhibit the same characteristics of nanoparticles inside the eyes [57]. The drugs which cannot be administered directly into the body, the nanoparticles were developed in those cases with the different route of administration. The used materials for the nanoparticle need to be biocompatible with the formulation. There are a different number of excipients those are been approved by the Food and Drug Administration (FDA) for the human use which needs to meet the needs of different polymers such as polylactic-co-glycolic acid, poly (ethylene glycol), and poly (hydroxy alkenoates). Moreover, bovine serum albumin, chitosan, Eudragit RS, and polyacrylic acid have been used for ocular drug delivery [58].

To improve the growing concept, its toxicity and potential of the nanoparticles need to be encountered with different materials but have several advantages over it [59]. There are different published articles which are based on the toxic or nontoxic studies of the drug to exhibit the toxicity associated and to identify it. Later on, it was reported that increased doses of cyclodextrin siRNA nanoparticle by intravenous injection have led to multiple tissue toxicities [60].

\section{THERAPEUTIC INVENTIONS OF DIFFERENT DRUGS}

There have been employed different therapies for the development of DR which have undergone clinical trials. Some of them are mentioned below [61].

\section{VEGF}

One of the main roles in the treatment of DR and DME, they are used for treating different pathological pathways in case of VEGF. It is considered as one of the potent mitogens, identified in 1989 [62]. The mechanism by which their releases have been noted by the cytokine of a huge number of different cells showing a different response to hypoxic conditions, and it was considered and was found to drastically increase in patients with PDR [63]. VEGF-mediated vascular growth is usually considered with the relief of hypoxia and it is considered as the most crucial process which is used in the treatment and development of vasculogenic and angiogenesis, they also allow them for the effective use and uniform retinal vascular architecture [64].

There different reported diseases factors in case of diseases like DR/DME, its release is calculated based on the pathological angiogenesis that is unresponsively present all over and features poorly constructed vessels that are prone to leak, they also cause different fluid accumulation within the retina. These are considered in different situations as vascular leakage is the most considered risk factor for DME, and they also found to be a better predictor for different clinical considerations of DME [65].

At the beginning of 2004, there were different series of anti-VEGF treatment which was granted by the FDA based on the different clinical trials carried out [66]. The different drugs considered are pegaptanib in 2004, ranibizumab in 2006, and the same for macular degeneration in 2015, and aflibercept in 2011. The main important Phase III RISE/RIDE trials have been reported as a gradual enhancement in the visual acuity in $\sim 45 \%$ of patients with DR/DME in relative to the anti-VEGF treatment. There is different advancement to be considered in case of the topical administration of the molecule that inhibits the progress with an ongoing preliminary clinical trial involving Pan Optica's PAN-90806. In addition, multiple clinical trials have been conducted or are currently in progress, examining combined anti-VEGF, and laser therapy approaches [67].

There are different nanotechnological advancement in many biomedical applications, such as gene therapy, drug delivery, and novel drug discovery techniques [68]. Nanoparticles have many milestone histories and also increase in the drug administration alone or to classic delivery systems, namely:

1. Sustained drug delivery of particular nanoparticle

2. Targeted delivery to specific cells or tissues; and

3. Reduced side effects they can bypass biological barriers, and especially blood-neural barriers, including the BRB and the blood-brain barrier $[69,70]$.

There are different ways for both therapy and ongoing diagnosis imaging functions and delivers the right drug at the right time and with the right dose. Some of the properties have been bought in different candidates with an improved way of increasing the hopes which are commonly called as "theranostics," which is a medical and technological fairly young field that is developing incredibly fast [71].

The dexamethasone which shows the sustained drug delivery from the nano micelles which is used for in vitro transscleral iontophoresis has been investigated using a mixed micellar solution constituted by simple (SSM) and mixed micelles (SMM) constituted with sodium taurocholate alone or with egg lecithin [72].

\section{RAS INHIBITORS}

The RAS is one among the most important and considered way which is employed to regulator the blood pressure which leads to renal sodium reabsorption and vascular tone, it also considered as one of the target types of delivery for DR therapies at a different level. These involve, namely angiotensinogen, which is converted to angiotensin I, then angiotensin II by renin and angiotensin-converting enzyme also known as kininase II [73].

Angiotensin II is mostly used as a vasoconstrictor, and these also help in the secretion or release of aldosterone, which increases sodium reabsorption in the kidney. They are different approaches that need to be developed, and the studies are going to know the different measures and roles for RAS in angiogenesis are taken into consideration, this leads to 
the development of both animal studies and clinical trials investigating the therapeutic potential of RAS inhibitors in DR/DME [74].

The direct study of $>1400$ patients found that oral candesartan reduced retinal microaneurysm progression in both Types I and II diabetic patients but the therapy was not associated with increased DR regression, reduced DR progression, or decrease in the incidence of DME [75].

\section{NONSTEROIDAL ANTI-INFLAMMATORY DRUGS (NSAID)}

The ETDRS in different patients with the NPDR or mild PRD has been treated which leads to the advancement in the treatment of more severe PDR and visual impairment by prescribing patients with the daily dosage of an oral regimen of the NSAID mainly used among those are aspirin.

There are different studies which showed nearly 4000 patients take $650 \mathrm{mg} /$ day dose; this did not achieve the needs, subsequent clinical trials with other NSAIDs have successfully attenuated DR symptoms [76].

Ketorolac is used as an NSAID prostaglandin inhibitor, which is used for pain relief in most of the medical procedures these include pan-retinal photocoagulation [77]. They belong to the subclass of NSAIDs (coxibs) that target cyclo-oxygenase (COX) enzymes responsible for prostaglandin production [78].

The eye drops form of ketorolac (Acuvail, Allergan) is used mostly as additional single-center clinical trials to gauge intraocular concentrations of the drug and various inflammatory mediators including prostaglandins in patients with DR. In addition, both ketorolac and the coxib, nepafenac, have been investigated for different therapeutic intervention of DME. However, the recent DRCR protocol study failed, leading to reduction of the retinal volume in patients those who are been treated with topical nepafenac [79].

Coxib and diclofenac have been tested preoperatively in a single center trial for reduction of DME following cataract removal in patients with diabetes. Coxibs and specifically ketorolac have a history of occasional serious side effects [80].

\section{TREATMENTS FOR RETINAL DISEASES AND OCULAR BARRIERS}

Therapeutic agents such as monoclonal antibodies such as bevacizumab, fusion proteins such as aflibercept and small molecules are identified for the treatment of retinal diseases. All these molecules show higher specificity, and decreased toxicity than other antibodies, small molecule, etc. [81]. The anti-VEGF drugs, for example, bevacizumab (Avastin ${ }^{\circledR}$; Genentech, Inc., CA, USA), ranibizumab (Lucentis ${ }^{\circledR}$; Genentech, Inc., CA, USA), and aflibercept $\left(\right.$ Eylea $^{\circledR}$; Regeneron Pharmaceuticals, Inc., NY, USA), have been commonly used in US to treat "wet" (neovascular) AMD over the last decade [82].

Regarding DR, a retinal disease is been identified and reported to cause diabetes, in 2015 US FDA approved aflibercept employed for the different treatments, following aflibercept's earlier approval for treatment of wet AMD. On the other hand, to prevent vision loss, immunocompromised patients with cytomegalovirus retinitis often need long-term antiretroviral therapy (e.g.ganciclovir) [83]. It has been noted that the only two options of administering the drug into the patient's eye are intravitreal and subretinal injection. Multiple physical boundaries within the eye are one of the important considerations of this type of drug delivery, including corneal and conjunctival epithelium, blood-aqueous barriers, and BRB. These structures stop the passage of molecules and fluids to the retina and also resist drug penetration [84].

Most widely used methods for ophthalmic medication administration include conventional drug administration systems such as eye drops, suspensions, and ointments, but they mainly target the anterior segment of the eye [85]. The retinal treatment for the diseases, conventional dosage forms (i.e.eye drops) needs to traverse the anterior chamber before the drug is able to penetrate deeply into the posterior tissues. The corneal and conjunctival epithelium is the main barriers of the anterior chamber. Besides those physical barriers, other anatomical and physiological processes, such as reflex blinking, tear turnover, and nasolacrimal drainage cause decrease in the ocular bioavailability of the drug. Hence, topical installation is rarely used in the treatment of retinal diseases since there is no exposure to the retina [86].

Topical gel systems were developed to increase drug retention in the cornea and conjunctiva by the pharmaceutical companies that prolong drug exposure, particularly for dry eye syndrome [87]. For systemic administration, BRB that includes capillary endothelial cells (inner BRB) and retinal pigment epithelial cells (retinal pigment epithelium cells, outer BRB) is the main obstacles for drug delivery due to the innate protective nature of the demarcation from the rest of the circulation [58].

\section{EMPLOYMENT OF NANOTECHNOLOGY IN OCULAR DRUG DELIVERY}

The nanomaterials are mainly interpreted and commonly used for the application of nanosized particles (1-1000 nm) in the area of pharmaceutical sciences [55]. The different nanoparticles used as ophthalmic agents to improve the poor solubility of the drugs and to target the retina of the eye which enhances the cellular uptake of drug and transport of drugs across the biological barriers and the residence time, and to protect the drug from degradation. There is a greater platform for designing the non-invasive system in the delivery of drug from the offer of nanotechnology-based therapy in a sustained manner [54].

There are different fields in the drug delivery and material science in the new advancement of the development of numerous nanomaterials with the remarkable development in numerous nanomaterials. There are different materials in the development of nanoparticles these include synthetic polymers such as micelles, dendrimers, hydrogel, liposomes, proteins, and inorganic compounds [56].

It is lipid bi-layered small vesicles that have both hydrophilic and lipophilic drugs those are called as Liposomes. e pharmacokinetic and pharmacodynamics improvement of the liposomes formulation with the sustained release of the medication to target the tissues and also with less systemic toxicity [88]. There needs to have special attention with the size, shape, materials, and surface properties of nanoparticles for effectively utilized in ocular drug delivery. Size should be managed to prevent rapid leakage into the blood vessels that penetrate the biological barriers into the retina [89].

CONCLUSION

There are not many formulations developed for this purpose. Effective measures were not taken for the development of the treatment of macular edema or the DR. As reviewed, it is found that there is different drug formulation such as RAS, VGF, and NSAIDS which were used for the betterment of the disease but it was found that nanotechnology-based nanoparticles employed showed effective results when compared to all other types of treatments. Due to the surface area, size, and the ability to cross the barriers, it is the best advancement for the treatment of the disease.

\section{AUTHORS' CONTRIBUTIONS}

All the authors have contributed equally in the design, development, review, and finalization of the contents of the manuscript.

\section{CONFLICTS OF INTEREST}

The authors confirm that this article content has no conflicts of interest.

\section{REFERENCES}

1. Zhang J, Liu R, Kuang HY, Gao XY, Liu HL. Protective treatments and their target retinal ganglion cells in diabetic retinopathy. 
Brain Res Bull 2017;132:53-60.

2. Pal S, Gautam S, MishraA, Maurya R, SrivastavaAK. Antihyperglycemic and antidyslipidemic potential of Ipomoea batatas leaves in validated diabetic animal models. Int J Pharm Pharm Sci 2015;7:176-86.

3. Salwe KJ, Sachdev DO, Bahurupi Y, Kumarappan M. Evaluation of antidiabetic, hypolipedimic and antioxidant activity of hydroalcoholic extract of leaves and fruit peel of Punica granatum in male wistar albino rats. J Nat Sci Biol Med 2015;6:56-62.

4. Madsen-Bouterse SA, Kowluru RA. Oxidative stress and diabetic retinopathy: Pathophysiological mechanisms and treatment perspectives. Rev Endocr Metab Disord 2008;9:315-27.

5. Kim J, Moon E, Kim TH. Successful midterm management with an herbal decoction, modified-Goshajinkigan (mGJG) for non-proliferative diabetic retinopathy: A case study. Explore (NY) 2018;14:295-9.

6. Nakao S, Yoshida S, Sonoda KH. An overview of diabetic retinopathy. In: Nutritional and Therapeutic Interventions for Diabetes and Metabolic Syndrome. $2^{\text {nd }}$ ed. London: Academic Press, An Imprint of Elsevier; 2018. p. 139-54.

7. Yau JW, Rogers SL, Kawasaki R, Lamoureux EL, Kowalski JW, Bek T, et al. Global prevalence and major risk factors of diabetic retinopathy. Diabetes Care 2012;35:556-64.

8. Fong DS, Aiello L, Gardner TW, King GL, Blankenship G, Cavallerano JD, et al. Retinopathy in diabetes. Diabetes Care 2004;27 Suppl 1:S84-7.

9. Hernández C, Simó-Servat O, Simó R. Somatostatin and diabetic retinopathy: Current concepts and new therapeutic perspectives. Endocrine 2014;46:209-14

10. Ciulla TA, Amador AG, Zinman B. Diabetic retinopathy and diabetic macular edema: Pathophysiology, screening, and novel therapies. Diabetes Care 2003;26:2653-64.

11. Kane FE, Burdan J, Cutino A, Green KE. Iluvien: A new sustained delivery technology for posterior eye disease. Expert Opin Drug Deliv 2008;5:1039-46.

12. Fangueiro JF, Silva AM, Garcia ML, Souto EB. Current nanotechnology approaches for the treatment and management of diabetic retinopathy. Eur J Pharm Biopharm 2015;95:307-22.

13. Uchegbu IF, Schätzlein AG, Cheng WP, Lalatsa A, editors. Fundamentals of Pharmaceutical Nanoscience. New York: Springer Science \& Business Media; 2013.

14. Rosberger DF. Diabetic retinopathy: Current concepts and emerging therapy. Endocrinol Metab Clin North Am 2013;42:721-45.

15. Goldberg MF, Fine SL, editors. Symposium on the Treatment of Diabetic Retinopathy. Arlington, Va.: US Neurological and Sensory Disease Control Program; 1969.

16. Gupta A, Chhikara R. Diabetic retinopathy: Present and past. Procedia Comput Sci 2018;132:1432-40

17. Agurto C, Murray V, Barriga E, Murillo S, Pattichis M, Davis H, et al. Multiscale AM-FM methods for diabetic retinopathy lesion detection. IEEE Trans Med Imaging 2010;29:502-12.

18. Bitirgen G, Ozkagnici A, Malik RA, Kerimoglu H. Corneal nerve fibre damage precedes diabetic retinopathy in patients with type 2 diabetes mellitus. Diabet Med 2014;31:431-8.

19. Petropoulos IN, Green P, Chan AW, Alam U, Fadavi H, Marshall A, et al. Corneal confocal microscopy detects neuropathy in patients with type 1 diabetes without retinopathy or microalbuminuria. PLoS One 2015;10:e123517.

20. Srinivasan S, Dehghani C, Pritchard N, Edwards K, Russell AW, Malik RA, et al. Ophthalmic and clinical factors that predict four-year development and worsening of diabetic retinopathy in type 1 diabetes. J Diabetes Complications 2018;32:67-74.

21. Tan NYQ, Tham YC, Ding Y, Yasuda M, Sabanayagam C, Saw SM, et al. Associations of peripapillary atrophy and fundus tessellation with diabetic retinopathy. Ophthalmol Retina 2018;2:574-81.

22. Wong TY, Klein R, Islam FM, Cotch MF, Folsom AR, Klein BE, et al. Diabetic retinopathy in a multi-ethnic cohort in the United States. Am J Ophthalmol 2006;141:446-55.

23. Grading diabetic retinopathy from stereoscopic color fundus photographs - an extension of the modified airlie house classification. ETDRS report number 10. Early Treatment Diabetic Retinopathy Study Research Group. Ophthalmology 1991;98:786-806

24. Kempen JH, O'Colmain BJ, Leske MC, Haffner SM, Klein R, Moss SE, et al. The prevalence of diabetic retinopathy among adults in the United States. Arch Ophthalmol 2004;122:552-63.

25. Royle P, Mistry H, Auguste P, Shyangdan D, Freeman K, Lois N, et al. Pan-retinal photocoagulation and other forms of laser treatment and drug therapies for non-proliferative diabetic retinopathy: Systematic review andeconomic evaluation. Health Technol Assess 2015;19:v-xxviii, 1-247.
26. Photocoagulation treatment of proliferative diabetic retinopathy. Clinical application of diabetic retinopathy study (DRS) findings, DRS report number 8 . The Diabetic Retinopathy Study Research Group. Ophthalmology 1981;88:583-600.

27. Lövestam-Adrian M, Agardh CD, Torffvit O, Agardh E. Type 1 diabetes patients with severe non-proliferative retinopathy may benefit from panretinal photocoagulation. Acta Ophthalmol Scand 2003;81:221-5.

28. Stenkula S. Photocoagulation in diabetic retinopathy. A multicentre study in Sweden. Acta Ophthalmol Suppl 1984;162:1-100.

29. Funatsu H, Yamashita H. Pathogenesis of diabetic retinopathy and the renin-angiotensin system. Ophthalmic Physiol Opt 2003;23:495-501.

30. Bresnick GH. Diabetic macular edema. A review. Ophthalmology 1986;93:989-97.

31. Helfgott A, Helfgott AE, Mullany S. Using mathematics to avoid blindness in diabetics. J Model Ophthalmol 2018;2:42-70.

32. Aiello LP, Avery RL, Arrigg PG, Keyt BA, Jampel HD, Shah ST, et al. Vascular endothelial growth factor in ocular fluid of patients with diabetic retinopathy and other retinal disorders. N Engl J Med 1994;331:1480-7.

33. Stitt AW, Lois N, Medina RJ, Adamson P, Curtis TM. Advances in our understanding of diabetic retinopathy. Clin Sci (Lond) 2013;125:1-7.

34. Shepro D, Morel NM. Pericyte physiology. FASEB J 1993; 7:1031-8.

35. Fangueiro JF, Andreani T, Egea MA, Garcia ML, Souto SB, Silva AM, et al. Design of cationic lipid nanoparticles for ocular delivery: Development, characterization and cytotoxicity. Int J Pharm 2014;461:64-73.

36. Nathan DM; DCCT/EDIC Research Group. The diabetes control and complications trial/epidemiology of diabetes interventions and complications study at 30 years: Overview. Diabetes Care 2014;37:9-16.

37. Porta M, Bandello F. Diabetic retinopathy: A clinical update. Diabetologia 2002;45:1617-34

38. Beulens JW, Patel A, Vingerling JR, Cruickshank JK, Hughes AD, Stanton A, et al. Effects of blood pressure lowering and intensive glucose control on the incidence and progression of retinopathy in patients with type 2 diabetes mellitus: A randomised controlled trial. Diabetologia 2009;52:2027-36

39. Progression of retinopathy with intensive versus conventional treatment in the diabetes control and complications trial. Diabetes control and complications trial research group. Ophthalmology 1995;102:647-61.

40. Intensive blood-glucose control with sulphonylureas or insulin compared with conventional treatment and risk of complications in patients with type 2 diabetes (UKPDS 33). UK Prospective Diabetes Study (UKPDS) Group. Lancet 1998;352:837-53.

41. Matthews DR, Stratton IM, Aldington SJ, Holman RR, Kohner EM; UK Prospective Diabetes Study Group. Risks of progression of retinopathy and vision loss related to tight blood pressure control in type 2 diabetes mellitus: UKPDS 69. Arch Ophthalmol 2004;122:1631-40.

42. Indications for photocoagulation treatment of diabetic retinopathy: Diabetic retinopathy study report no. 14. The Diabetic Retinopathy Study Research Group. Int Ophthalmol Clin 1987;27:239-53.

43. Early photocoagulation for diabetic retinopathy. ETDRS report number 9. Early Treatment Diabetic Retinopathy Study Research Group. Ophthalmology 1991;98:766-85.

44. Tzekov R, Arden GB. The electroretinogram in diabetic retinopathy. Surv Ophthalmol 1999;44:53-60.

45. Waring GO $3^{\text {rd }}$, Rodrigues MM. Patterns of pathologic response in the cornea. Surv Ophthalmol 1987;31:262-6.

46. Javitt JC, Aiello LP. Cost-effectiveness of detecting and treating diabetic retinopathy. Ann Intern Med 1996;124:164-9.

47. Klein R, Klein BE, Moss SE, Davis MD, DeMets DL. The Wisconsin epidemiologic study of diabetic retinopathy. VI. Retinal photocoagulation. Ophthalmology 1987:94:747-53.

48. Diabetes Control and Complications Trial Research Group, Nathan DM, Genuth S, Lachin J, Cleary P, Crofford O. The effect of intensive treatment of diabetes on the development and progression of long-term complications in insulin-dependent diabetes mellitus. N Engl J Med 1993;329:977-86.

49. Stewart MW. Treatment of diabetic retinopathy: Recent advances and unresolved challenges. World J Diabetes 2016;7:333-41.

50. Sinawat S, Rattanapakorn T, Sanguansak T, Yospaiboon Y, Sinawat S. Intravitreal bevacizumab for proliferative diabetic retinopathy with new dense vitreous hemorrhage after full panretinal photocoagulation. Eye (Lond) 2013;27:1391-6.

51. Figueira J, Silva R, Henriques J, Caldeira Rosa P, Laíns I, Melo $\mathrm{P}$, et al. Ranibizumab for high-risk proliferative diabetic retinopathy: An exploratory randomized controlled trial. Ophthalmologica 2016;235:34-41.

52. Gross JG, Glassman AR, Liu D, Sun JK, Antoszyk AN, Baker CW, et al. Five-year outcomes of panretinal photocoagulation vs. intravitreous 
ranibizumab for proliferative diabetic retinopathy: A randomized clinical trial. JAMA Ophthalmol 2018;136:1138-48.

53. Bisht R, Mandal A, Jaiswal JK, Rupenthal ID. Nanocarrier mediated retinal drug delivery: Overcoming ocular barriers to treat posterior eye diseases. Wiley Interdiscip Rev Nanomed Nanobiotechnol 2018;10:1-21.

54. Jiang S, Franco YL, Zhou Y, Chen J. Nanotechnology in retinal drug delivery. Int J Ophthalmol 2018;11:1038-44.

55. Duncan R, Gaspar R. Nanomedicine(s) under the microscope. Mol Pharm 2011;8:2101-41.

56. Cho K, Wang X, Nie S, Chen ZG, Shin DM. Therapeutic nanoparticles for drug delivery in cancer. Clin Cancer Res 2008;14:1310-6.

57. Koo H, Moon H, Han H, Na JH, Huh MS, Park JH, et al. The movement of self-assembled amphiphilic polymeric nanoparticles in the vitreous and retina after intravitreal injection. Biomaterials 2012;33:3485-93.

58. Nakhlband A, Barar J. Impacts of nanomedicines in ocular pharmacotherapy. Bioimpacts 2011;1:7-22.

59. El-Ansary A, Al-Daihan S, Bacha AB, Kotb M. Toxicity of novel nanosized formulations used in medicine. In: Oxidative Stress and Nanotechnology. Totowa, NJ: Humana Press; 2013. p. 47-74.

60. Heidel JD, Yu Z, Liu JY, Rele SM, Liang Y, Zeidan RK, et al. Administration in non-human primates of escalating intravenous doses of targeted nanoparticles containing ribonucleotide reductase subunit M2 siRNA. Proc Natl Acad Sci U S A 2007;104:5715-21.

61. Keck PJ, Hauser SD, Krivi G, Sanzo K, Warren T, Feder J, et al. Vascular permeability factor, an endothelial cell mitogen related to PDGF. Science 1989;246:1309-12.

62. Leung DW, Cachianes G, Kuang WJ, Goeddel DV, Ferrara N. Vascular endothelial growth factor is a secreted angiogenic mitogen. Science 1989;246:1306-9.

63. Provis JM. Development of the primate retinal vasculature. Prog Retin Eye Res 2001;20:799-821.

64. Zhang $X$, Liu W, Wu S, Jin J, Li W, Wang N. Calcium dobesilate for diabetic retinopathy: A systematic review and meta-analysis. Sci China Life Sci 2015;58:101-7.

65. Sander B, Thornit DN, Colmorn L, Strøm C, Girach A, Hubbard LD, et al. Progression of diabetic macular edema: Correlation with blood retinal barrier permeability, retinal thickness, and retinal vessel diameter. Invest Ophthalmol Vis Sci 2007;48:3983-7.

66. Murakami T, Felinski EA, Antonetti DA. Occludin phosphorylation and ubiquitination regulate tight junction trafficking and vascular endothelial growth factor-induced permeability. J Biol Chem 2009;284:21036-46.

67. Nguyen QD, Brown DM, Marcus DM, Boyer DS, Patel S, Feiner L, et al. Ranibizumab for diabetic macular edema: Results from 2 phase III randomized trials: RISE and RIDE. Ophthalmology 2012;119:789-801.

68. Sahoo SK, Dilnawaz F, Krishnakumar S. Nanotechnology in ocular drug delivery. Drug Discov Today 2008;13:144-51.

69. Fangueiro JF. Cationic Lipid Nanomedicines for the Treatment of Diabetic Retinopathy (Doctoral Dissertation); 2016

70. Jo DH, Kim JH, Lee TG, Kim JH. Size, surface charge, and shape determine therapeutic effects of nanoparticles on brain and retinal diseases. Nanomedicine 2015;11:1603-11.
71. Kelkar SS, Reineke TM. Theranostics: Combining imaging and therapy. Bioconjug Chem 2011;22:1879-903.

72. Chopra P, Hao J, Li SK. Sustained release micellar carrier systems for iontophoretic transport of dexamethasone across human sclera. J Control Release 2012;160:96-104.

73. Wegman-Ostrosky T, Soto-Reyes E, Vidal-Millán S, Sánchez-Corona J. The renin-angiotensin system meets the hallmarks of cancer. J Renin Angiotensin Aldosterone Syst 2015; 16:227-33.

74. Vincent F, Bonnin P, Clemessy M, Contrerès JO, Lamandé N, Gasc JM, et al. Angiotensinogen delays angiogenesis and tumor growth of hepatocarcinoma in transgenic mice. Cancer Res 2009;69:2853-60.

75. Sjølie AK, Klein R, Porta M, Orchard T, Fuller J, Parving HH, et al. Retinal microaneurysm count predicts progression and regression of diabetic retinopathy. Post-hoc results from the DIRECT programme. Diabet Med 2011;28:345-51.

76. Effects of aspirin treatment on diabetic retinopathy. ETDRS report number 8. Early Treatment Diabetic Retinopathy Study Research Group. Ophthalmology 1991;98:757-65.

77. Esgin H, Samut HS. Topical ketorolac $0.5 \%$ for ocular pain relief during scatter laser photocoagulation with $532 \mathrm{~nm}$ green laser. J Ocul Pharmacol Ther 2006;22:460-4.

78. Patrício JP, Barbosa JP, Ramos RM, Antunes NF, de Melo PC. Relative cardiovascular and gastrointestinal safety of non-selective non-steroidal anti-inflammatory drugs versus cyclo-oxygenase-2 inhibitors: Implications for clinical practice. Clin Drug Investig 2013;33:167-83.

79. Friedman SM, Almukhtar TH, Baker CW, Glassman AR, Elman MJ, Bressler NM, et al. Topical nepafenec in eyes with noncentral diabetic macular edema. Retina 2015;35:944-56.

80. Bolinger MT, Antonetti DA. Moving past anti-VEGF: Novel therapies for treating diabetic retinopathy. Int J Mol Sci 2016;17. pii: E1498.

81. Zafir-Lavie I, Michaeli Y, Reiter Y. Novel antibodies as anticancer agents. Oncogene 2007;26:3714-33.

82. Yonekawa Y, Kim IK. Clinical characteristics and current treatment of age-related macular degeneration. Cold Spring Harb Perspect Med 2014;5:a017178.

83. Vadlapudi AD, Vadlapatla RK, Mitra AK. Current and emerging antivirals for the treatment of cytomegalovirus (CMV) retinitis: An update on recent patents. Recent Pat Antiinfect Drug Discov 2012;7:8-18.

84. Del Amo EM, Urtti A. Current and future ophthalmic drug delivery systems. A shift to the posterior segment. Drug Discov Today 2008; $13: 135-43$

85. Kim YC, Chiang B, Wu X, Prausnitz MR. Ocular delivery of macromolecules. J Control Release 2014;190:172-81.

86. Xu Q, Kambhampati SP, Kannan RM. Nanotechnology approaches for ocular drug delivery. Middle East Afr J Ophthalmol 2013;20:26-37.

87. Lavik E, Kuehn MH, Kwon YH. Novel drug delivery systems for glaucoma. Eye (Lond) 2011;25:578-86.

88. Deshpande PP, Biswas S, Torchilin VP. Current trends in the use of liposomes for tumor targeting. Nanomedicine (Lond) 2013;8:1509-28

89. Kim TW, Lindsey JD, Aihara M, Anthony TL, Weinreb RN. Intraocular distribution of 70-kDa dextran after subconjunctival injection in mice. Invest Ophthalmol Vis Sci 2002;43:1809-16. 